\title{
Micronageurs naturels et artificiels
}

Le plancton ou les colonies de bactéries sont des exemples de collection d'entités de petite taille capables de se mouvoir de façon autonome et cohérente : on parle de " micronageurs ". Leur comportement a inspiré les physiciens, qui ont cherché à en rationaliser la phénoménologie. Nous avons donc décidé de regrouper dans un dossier plusieurs articles qui illustrent les comportements riches et inhabituels des micronageurs.

L'idée d'une vie indécelable présente aux échelles microscopiques et capable d'envahir le corps humain remonte à plus de vingt-six siècles. Elle apparaît pour la première fois en Inde dans les écritures de Jain. Cette croyance se renforce aussi en Occident jusqu'au Moyen Âge, où les premières théories de la contagion reposent sur le transport de minuscules créatures entre nos corps. L'existence définitive des micro-organismes a été établie dès 1675 par Antonie Van Leeuwenhoek [1] qui, en concevant un microscope pour inspecter la qualité de ses étoffes, découvre les premiers mouvements de ce qu'il décrivait comme des essaims de vers et d'anguilles microscopiques agitant l'eau dans laquelle ils évoluent, lui donnant l'aspect d'un « liquide vivant »[2]. Van Leeuwenhoek découvrait

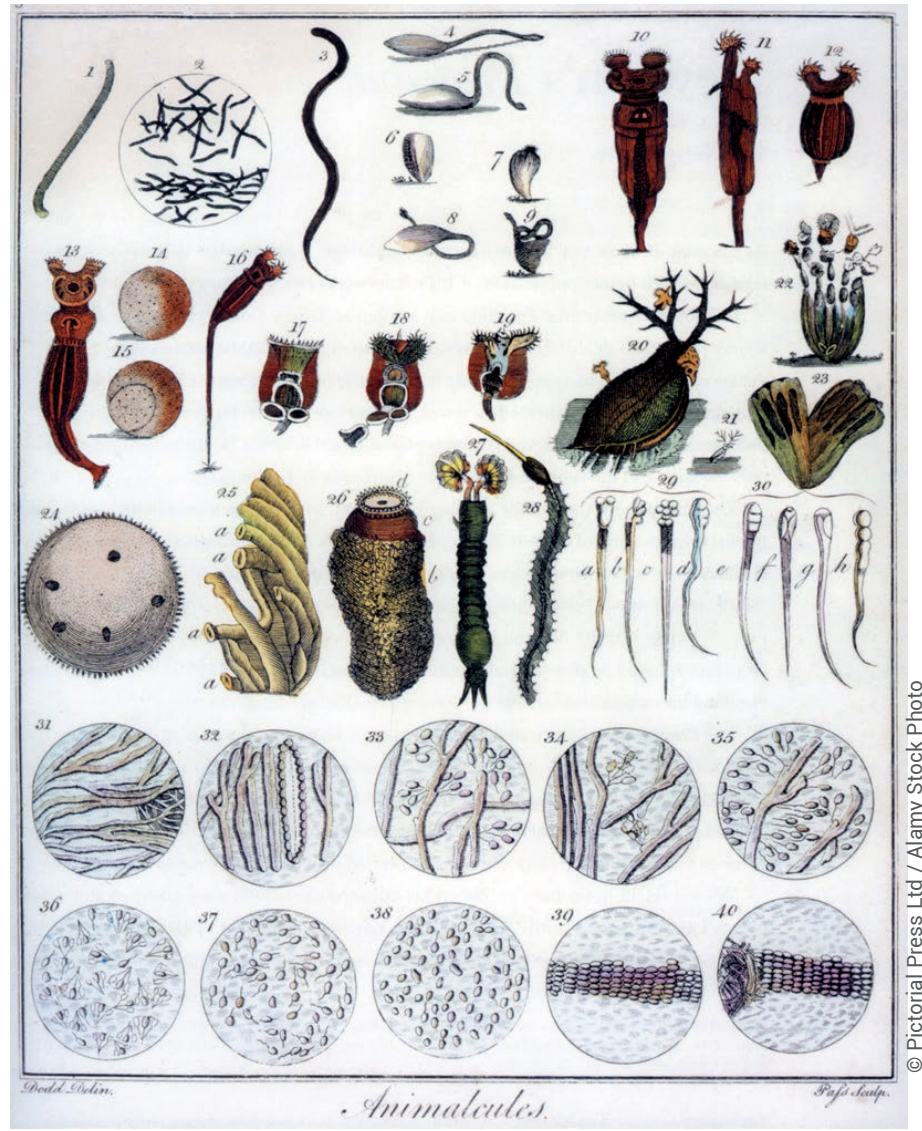

les premiers micronageurs. En dépit du développement constant de la microbiologie, ce n'est qu'au milieu du $20^{\mathrm{e}}$ siècle que les physiciens purent décrire la locomotion de microstructures déformables incapables d'exploiter leur inertie pour se propulser. Aux petites échelles, les forces visqueuses stoppent instantanément un corps qui cesse de se déformer. C'est finalement au début des années 2000 que les travaux conjoints des biologistes et des physiciens permirent de comprendre les mouvements de ces micro-organismes qui ont évolué ou perduré, en ne limitant s'est vue être confirmée ces dix dernières années par des mesures quantitatives sur des nuées d'oiseaux et des essaims d'insectes. Ainsi, en utilisant des concepts et des outils de la physique de la matière condensée et de la physique statistique, une ligne de recherche importante se consacre à décrire des tissus cellulaires, des biofilms, des suspensions bactériennes et le cytosquelette intracellulaire comme des matériaux actifs obéissant à d'autres lois de conservation, montrant des dynamiques inaccessibles aux systèmes passifs. 


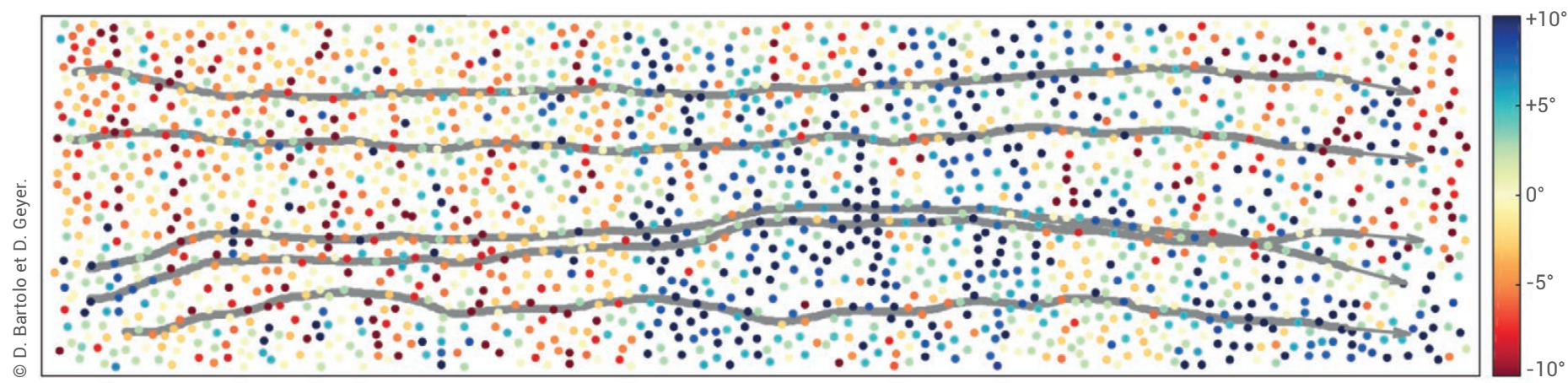

1. Assemblée de colloïdes autopropulsés formant un liquide capable de s'écouler spontanément. La couleur des colloïdes indique l'orientation de leur vitesse par rapport à l'axe horizontal (angle en degrés). Les trajectoires de cinq colloïdes localisés à l'extrémité droite des lignes grises illustrent leur mouvement dirigé de la gauche vers la droite de l'image. Ces trajectoires sont mesurées pendant 0,9 s. Largeur de l'image : 1 mm.

\section{Les mécanismes de propulsion des micronageurs naturels}

Les micronageurs naturels ont deux stratégies pour se propulser à travers un fluide : soit ils poussent sur le fluide (ils sont alors appelés "pousseurs"), soit ils le tirent vers eux (ce sont des «tireurs »). L'article de H. Auradou et al. (p. 20) s'intéresse à des pousseurs, tandis que celui de $\mathrm{S}$. Rafaï et P. Peyla (p. 24) s'intéresse à des tireurs.

Les figures Ela,b illustrent la technique de nage des bactéries pousseuses Escherichia coli [i]. Ces bactéries possèdent entre cinq et dix flagelles, longs filaments hélicoïdaux de protéines connectés à leur corps par des moteurs cellulaires. Ceux-ci mettent en rotation les flagelles qui se groupent alors en une tresse hélicoïdale soumise à une force propulsive orientée vers l'avant, laquelle propulse la bactérie et crée un écoulement du fluide vers l'arrière. Le corps, soumis à un couple de réaction opposé à celui appliqué à l'hélice, tourne en sens opposé. En avançant, la bactérie pousse le fluide, crée ainsi un écoulement vers l'avant et est soumise par réaction à une force de trainée dirigée vers l'arrière. L'effet de la bactérie sur le fluide à une distance grande devant sa taille se modélise donc par deux forces opposées en régime stationnaire pour avoir une résultante nulle et de points d'application différents : ce dipôle de forces induit autour du micro-organisme l'écoulement schématisé dans la figure E1b. Dans l'axe de la bactérie, le fluide est éjecté vers l'extérieur, aussi bien à l'avant qu'à l'arrière. II est au contraire attiré vers la bactérie dans les directions perpendiculaires.

Les figures E1c,d correspondent à des microalgues comme les Chlamydomonas reinhardtii qui sont, au contraire, un exemple de tireurs [ii] : elles tirent sur le fluide en utilisant des flagelles frontaux comme le ferait un nageur de brasse avec ses bras. À l'arrière, le fluide est ramené vers le nageur (figs. E1c,d). On a à nouveau un dipôle de forces, mais ses composantes sont en sens inverse des précédentes.

[i] H. C. Berg, E. coli in motion, Springer Science \& Business Media (2004).

[ii] M. Polin et al., Science 24 (2009) 487.
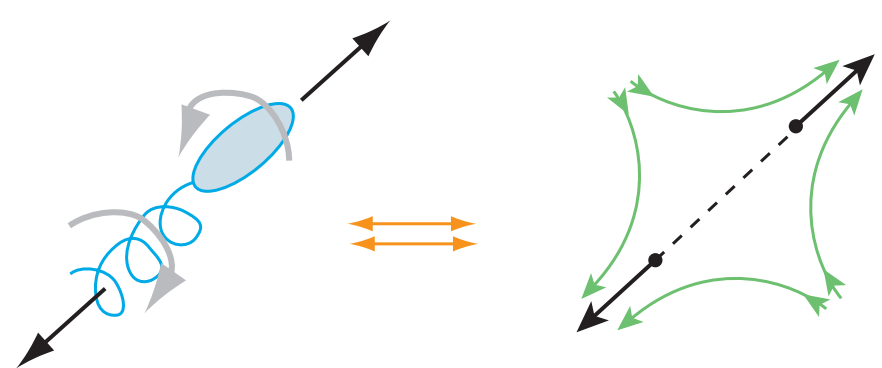

a

b
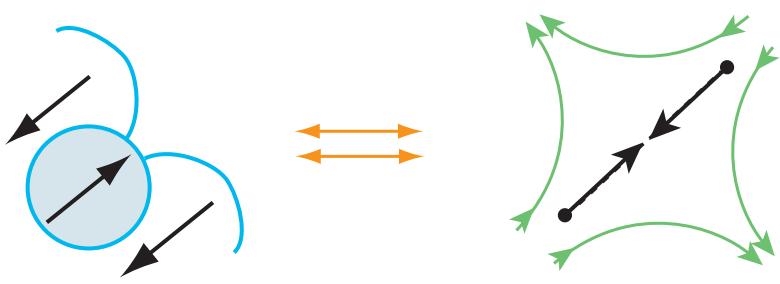

d

E1. Pousseurs et tireurs : deux façons de nager.

$(a, c)$ : Mouvements de nage (flèches grises) et forces induites (flèches noires) pour les micro-organismes pousseurs (a) et tireurs (c).

(b,d) : Dipôles de forces équivalents (vecteurs en noir) et écoulements du fluide provoqués par la nage (lignes vertes) pour ces microorganismes. 